\title{
Gravitational quantum limit for length measurements
}

\author{
Marc-Thierry Jaekel ${ }^{a}$ and Serge Reynaud ${ }^{b}$ \\ (a) Laboratoire de Physique Théorique de l'ENS * 24 rue Lhomond F'75231 Paris Cedex 05 France \\ (b) Laboratoire de Spectroscopie Hertzienne de l'ENS ${ }^{\dagger}$, 4 place Jussieu F75252 Paris Cedex 05 France
}

(Physics Letters A 185 (1994) 143-148)

\begin{abstract}
We discuss a limit for sensitivity of length measurements which is due to the effect of vacuum fluctuations of gravitational field. This limit is associated with irreducible quantum fluctuations of geodesic distances and it is characterized by a noise spectrum with an order of magnitude mainly determined by Planck length. The gravitational vacuum fluctuations may (in an analysis restricted to questions of principle and when the measurement strategy is optimized) dominate fluctuations added by the measurement apparatus if macroscopic masses, i.e. masses larger than Planck mass, are used.
\end{abstract}

\section{INTRODUCTION}

Quantum fluctuations put limits on the sensitivity in length measurements. For measurements based upon electromagnetic probes, sensitivity appears at first sight to be bound by the 'standard quantum limit', a compromise between length fluctuations on one hand and momentum fluctuations of end-points on the other hand [1]. Stated in other words, phase fluctuations of the electromagnetic probe appear as fluctuations of the measured length, while intensity fluctuations give rise to a random motion of the end-points (for instance mirrors of an interferometer), through the action of radiation pressure. Since phase and intensity are quantum conjugated variables with fluctuations bound by an Heisenberg inequality, a standard quantum limit is derived when considering their contributions as uncorrelated [2].

However, phase and intensity fluctuations are not independent noise sources, and it is feasible, in principle, to push sensitivity beyond standard quantum limit [3]. It turns out that sensitivity is limited only by dissipation in the mechanical admittance of end-points $\mid$ | . Focussing the attention upon questions of principle and putting discussion of experimental problems aside (thermal fluctuations, limitations for optical or mechanical finesses, control of fluctuations of the electromagnetic probe, ..), one is then led to consider fundamental dissipation mechanisms which cannot be bypassed. One such mechanism, the radiation pressure of vacuum fluctuations, leads to a sensitivity limit [5] which is of the order of the Compton wavelength $\lambda_{C}$ associated with the end-points' masses.

This result could be expected from a mere dimensional analysis using the Planck constant $\hbar$, the light velocity $c$ and the mass $m$. As soon as gravity is considered however, Planck units of mass and length may be defined ( $G$ is the Newton's gravitational constant):

$$
\begin{aligned}
& m_{p}=\sqrt{\frac{\hbar c}{G}} \approx 2.210^{-8} \mathrm{~kg} \\
& l_{P}=c t_{p}=\frac{c}{\omega_{p}}=\sqrt{\frac{\hbar G}{c^{3}}} \approx 1.610^{-35} \mathrm{~m}
\end{aligned}
$$

and sensitivity in length measurements is expected to be limited by Planck length (see for example [6]). This is in conflict with the analysis sketched above, since Planck length is larger than Compton wavelength for masses greater than Planck mass. The purpose of the present letter is to show that a limit of the order of Planck length indeed appears when quantum fluctuations of gravitational field are taken into account.

The phase of an electromagnetic probe registers curvature perturbations associated with classical gravitational waves. In particular, it can detect a stochastic background of gravitational waves ( [7] and references in) which may have been generated by various astrophysical or cosmological mechanisms ( [8] and references in). Here, we will consider only the effect of gravitational vacuum fluctuations, i.e. proper quantum fluctuations of gravitational field. These fluctuations may be derived (for frequencies smaller than Planck frequency) in a linearized theory of gravitation in the same way as vacuum fluctuations of other physical fields (see for instance [9]). We show in the following that

\footnotetext{
${ }^{*}$ Unité propre du CNRS associée à l'Ecole Normale Supérieure et à l'Université Paris-Sud

${ }^{\dagger}$ Unité de l'Ecole Normale Supérieure et de l'Université Pierre et Marie Curie associée du CNRS
} 
they limit the sensitivity of geodesic measurements to Planck length. It has to be noted in particular that, though small, these fluctuations dominate vacuum pressure fluctuations for masses greater than Planck mass. This change in dominant fluctuations supports the intuition that Planck mass might be considered as a natural borderline between microscopic and macroscopic domains.

\section{QUANTUM LIMITS IN A LENGTH MEASUREMENT}

For completeness, we summarize in the present section the main results known for quantum limits in length measurements performed with an electromagnetic probe. The standard quantum limit is characterized as an ultimate detectable length variation $\Delta q$ :

$$
\left(\Delta q^{2}\right)^{S Q L} \approx \frac{\hbar}{m} T
$$

where $T$ is the measurement time. It can be derived by considering that the endpoints' positions are measured at two times separated by an interval $T$ and noting that positions at different times do not commute [10]. This derivation is implicitly based upon too restrictive assumptions and it consequently does not lead to the ultimate limit 11.

To present a more detailed analysis of ultimate limits, it is appropriate to describe length fluctuations by a time correlation or a noise spectrum defined according to the prescription:

$$
\begin{aligned}
C_{q q}(t) & \equiv\langle q(t) q(0)\rangle-\langle q(t)\rangle\langle q(0)\rangle \\
& \equiv \int \frac{d \omega}{2 \pi} C_{q q}[\omega] e^{-i \omega t}
\end{aligned}
$$

Ascribing the noise to phase and intensity fluctuations of the electromagnetic probe [2], one obtains the standard quantum limit when considering their contributions as statistically uncorrelated. Assuming that the signal is monitored at frequencies where the end-points are nearly free, one thus gets the noise spectrum [⿰]:

$$
C_{q q}^{S Q L}[\omega] \approx \frac{\hbar}{m \omega^{2}}
$$

This corresponds to a noise energy per unit bandwidth of the order of $\hbar$. The variance $\Delta q^{2}$ may then be evaluated for a measurement bandwidth $\Delta \omega$ :

$$
\left(\Delta q^{2}\right)^{S Q L} \approx \frac{\hbar}{m} \frac{\Delta \omega}{2 \pi \omega^{2}}
$$

The expression (2) is recovered when $\frac{\Delta \omega}{2 \pi \omega^{2}}$ is interpreted as the detection time $T$.

Now, the contributions of phase and intensity fluctuations are linearly superimposed in the monitored signal, and the total noise can be reduced [3] by squeezing the appropriate field quadrature component (references on squeezing may be found in [12]). Assuming that the field fluctuations around the probe frequency can be squeezed at convenience, one demonstrates that sensitivity is limited only by the dissipative part of the mirrors' mechanical admittance [ 4 . The same analysis shows that standard quantum limit is determined by the reactive part, and is therefore revealing the inadequacies of a measurement strategy rather than ultimate quantum limitations. This conclusion is consistent with a general analysis of the effects of noise and dissipation in high-sensitivity measurements [13].

A lower bound for dissipation is set by radiation pressure of vacuum fields, characterized by a spectrum $C_{F F}$ for the force $F$ exerted upon the mirrors:

$$
C_{F F}[\omega]=\frac{\hbar^{2}}{3 \pi c^{2}} \omega^{3} \theta(\omega) \Phi[\omega]
$$

The dimensionless function $\Phi$ is equal to 1 for a perfect mirror in a model world with only one spatial dimension [14]. In four-dimensional space-time, diffraction effects are present and è depends on geometric characteristics of the mirror 15]. For a perfectly reflecting mirror with a characteristic dimension $\rho, \Phi$ is found to be greater than 1 for $\omega \rho \gg c$ and smaller than 1 for $\omega \rho \ll c$, the latter case corresponding to a more realistic situation ( $\omega$ is a mechanical frequency). Note that the function $\Phi$ be enhanced by resonance effects in a cavity [16].

As a result of these force fluctuations, the mirrors undergo a random motion characterized by a noise spectrum [5:

$$
C_{q q}[\omega]=\frac{C_{F F}[\omega]}{m^{2} \omega^{4}}
$$


This random motion determines a 'vacuum-pressure quantum limit' which lies far beyond standard quantum limit (凷), with an order of magnitude given by the Compton wavelength $\lambda_{C}$ :

$$
C_{q q}^{V Q L}[\omega]=\frac{\Phi}{3 \pi} \lambda_{C}^{2} \frac{\theta(\omega)}{\omega} \quad \lambda_{C}=\frac{\hbar}{m c}
$$

Except for the factor $\frac{\Phi}{3 \pi}$, these expressions may be infered from simple dimensional arguments. They represent the ultimate sensitivity in a length measurement which would only be limited by quantum fluctuations of a measurement apparatus build with masses $m$.

\section{QUANTUM FLUCTUATIONS OF SPACE-TIME CURVATURE}

As discussed in the introduction, the phaseshift of the electromagnetic probe between the two end-points registers modifications of the space-time curvature associated with gravitational fluctuations, including in particular quantum fluctuations. For gravitational wave detectors, such fluctuations constitute the quantum fluctuations of the monitored signal itself.

Gravity fluctuations are usually represented as fluctuations of the metric $h_{\mu \nu}$ written in a particular gauge $[7$ 9. In order to emphasize the irreducible character of these space-time fluctuations, we prefer here to write manifestly Lorentz-invariant and gauge-independent correlation functions for linearized Riemann curvature fluctuations $R_{\mu \nu \rho \sigma}$ (more details on their derivation from the graviton propagator may be found in [17]; $k$ is a 4 -wavevector, and $k_{0}$ the corresponding frequency; the spectrum $C[k]$ is the 4-dimensional Fourier transform of the space-time correlation function $C(x)$, defined as in eq.(3); $\eta_{\mu \nu}$ is the Minkowski metric tensor with a signature $\left.(+,-,-,-)\right)$ :

$$
\begin{aligned}
C_{R_{\mu \nu \rho \sigma} R_{\mu^{\prime} \nu^{\prime} \rho^{\prime} \sigma^{\prime}}}[k] & =16 \pi^{2} l_{P}^{2} \theta\left(k_{0}\right) \delta\left(k^{2}\right) \\
& \times\left\{\mathcal{R}_{\mu \nu \mu^{\prime} \nu^{\prime}} \mathcal{R}_{\rho \sigma \rho^{\prime} \sigma^{\prime}}+\mathcal{R}_{\mu \nu \rho^{\prime} \sigma^{\prime}} \mathcal{R}_{\rho \sigma \mu^{\prime} \nu^{\prime}}-\mathcal{R}_{\mu \nu \rho \sigma} \mathcal{R}_{\mu^{\prime} \nu^{\prime} \rho^{\prime} \sigma^{\prime}}\right\} \\
\mathcal{R}_{\mu \nu \rho \sigma} & =\frac{1}{2}\left(k_{\mu} k_{\rho} \eta_{\nu \sigma}+k_{\nu} k_{\sigma} \eta_{\mu \rho}-k_{\nu} k_{\rho} \eta_{\mu \sigma}-k_{\mu} k_{\sigma} \eta_{\nu \rho}\right)
\end{aligned}
$$

As classical gravitational waves, gravitational vacuum fluctuations are concentrated upon the light cone (lightlike wavevectors) and correspond to vanishing Einstein curvature. Note that equations (6) give only the lowest order contribution proportional to $l_{P}^{2}$ (equivalently to $G$ ) to curvature fluctuations. Higher order contributions exist, due in particular to gravity of vacuum stress-tensor fluctuations. Their evaluation would in principle require a fully consistent theory of Quantum Gravity. They have however a much smaller magnitude when evaluated at frequencies much lower than Planck frequency $\omega_{P}$ (they scale at least as $l_{P}^{4}$ ), so that we shall ignore them here.

For simplicity, equations (6) and subsequent computations are written with natural space-time units $(c=1)$ and light velocity is reintroduced in the final expressions.

\section{QUANTUM FLUCTUATIONS OF GEODESIC DISTANCES}

As freely falling end-points follow geodesics, we will evaluate variations of the geodesic distance (measured as an electromagnetic phaseshift) by using the law of geodesic deviation [18]. Such a law gives the 'equivalent tidal acceleration' between points on two neighbouring geodesics as the product of their distance by the curvature component $R_{0 \mu 0 \nu} u^{\mu} u^{\nu}$ where $u$ is the electromagnetic wavevector (normalized in such a manner that $u_{0}=1$ ). In the general case of a finite distance between the two end-points, the geodesic deviation is obtained as an integral along the path of the probe.

For a measurement on a 'one-way track', the variation q of geodesic distance due to curvature fluctuations is obtained from ( $x$ is the 4-coordinate of the receiver, $t=x_{0}$ the corresponding time, $\tau$ the time of propagation from the emitter to the receiver, $\sigma$ the affine parameter along the path):

$$
\frac{d^{2} q}{d t^{2}}=\int_{0}^{\tau} R_{0 \mu 0 \nu}(x-u \sigma) u^{\mu} u^{\nu} d \sigma
$$

This relation is often written with $q(t)$ expressed in terms of metric components (usually in the transverse-traceless gauge) or with the redshift parameter $\left(-\frac{d q}{d t}\right)$ expressed in terms of time derivatives of such metric components (see [7] and references in; also compare with the Sachs-Wolfe formula [19]). 
After a translation into the frequency domain, one obtains the noise spectrum $C_{q q}$ describing the fluctuations of geodesic distance as an integral over gravitational wavevectors. Using the expressions (6) describing the gravitational vacuum fluctuations, one then derives a 'gravitational quantum limit':

$$
C_{q q}^{G Q L}[\omega]=l_{P}^{2} \frac{\theta(\omega)}{\omega} b[\omega]
$$

where the dimensionless function $b$ (denoted $b^{(1)}$ for one-way tracking) is:

$$
b^{(1)}[\omega]=\left\langle(1+\gamma)^{2}\left|e^{i \omega \tau}-e^{i \gamma \omega \tau}\right|^{2}\right\rangle
$$

Here, $\gamma$ is the cosine of the spatial angle between gravitational wavevector $k$ and electromagnetic propagation direction $u\left(\gamma=\frac{k_{m} u^{m}}{k_{0}}\right.$; note that $\left.\omega=k_{0}\right)$ and the symbol $<>$ represents an angular average (i.e. the average over $\gamma$ uniformly distributed between -1 and 1 ) so that:

$$
b^{(1)}[\omega]=\frac{8}{3}-\frac{4}{(\omega \tau)^{2}}+\frac{2 \sin (2 \omega \tau)}{(\omega \tau)^{3}}
$$

In the case of 'two-way tracking', the distance is measured as half a round trip time, which leads to a different expression for the function $b$ :

$$
\begin{aligned}
b^{(2)}[\omega] & =\frac{1}{4}\left\langle\left|(1+\gamma)\left(e^{i \omega \tau}-e^{i \gamma \omega \tau}\right)-(1-\gamma)\left(e^{-i \omega \tau}-e^{i \gamma \omega \tau}\right)\right|^{2}\right\rangle \\
& =1-\frac{\cos (2 \omega \tau)}{3}-\frac{3+\cos (2 \omega \tau)}{(\omega \tau)^{2}}+\frac{2 \sin (2 \omega \tau)}{(\omega \tau)^{3}}
\end{aligned}
$$

At the low-frequency limit (propagation length smaller than gravitational wavelength), the same expression is obtained in both cases of one-way and two-way tracking (a local tidal acceleration is measured in this case):

$$
\begin{aligned}
& b^{(1)}[\omega] \approx b^{(2)}[\omega] \approx(\omega \tau)^{2}\left\langle\left(1-\gamma^{2}\right)^{2}\right\rangle \quad(\omega \tau \ll 1) \\
& C_{q q}^{G Q L}[\omega] \approx \frac{8}{15} l_{P}^{2} \tau^{2} \omega \theta(\omega)
\end{aligned}
$$

At this limit, geodesic fluctuations may be reproduced by fluctuations of a local length scale, i.e. by conformal metric fluctuations. However, this picture is no longer valid for measurements involving several propagation directions. For example, in the geometry of a Michelson interferometer with two propagation directions, correlation functions depend on the angle between the two propagation directions (see [8] and references in).

At the high-frequency limit in contrast (propagation length larger than gravitational wavelength), fluctuations are found to be independent of the propagation time [20]:

$$
\begin{aligned}
C_{q q}^{G Q L}[\omega] & \approx l_{P}^{2} \frac{\theta(\omega)}{\omega} b[\infty] \quad(\omega \tau \gg 1) \\
b^{(1)}[\infty] & =\frac{8}{3} \quad b^{(2)}[\infty]=1
\end{aligned}
$$

At this limit, fluctuations of geodesic distances may be reproduced by independent stochastic motions of the two end-point positions.

\section{COMMUTATION RELATIONS FOR GEODESIC DISTANCE}

It has to be noted that the functions $b$ appearing in equation (8) are obtained in the same manner for any isotropic spectrum of gravitational fluctuations (see [] and references in) while the other factors appearing in expression (8) are characteristic of vacuum fluctuations. In particular, equations (8) describe fluctuations of non-commuting variables, as the curvature fluctuations (6) from which they are deduced (through the law of geodesic deviation (7)). This is made more visible by writing the commutator:

$$
[q(t), q(0)]=C_{q q}(t)-C_{q q}(-t)
$$


or, in the spectral domain:

$$
C_{q q}[\omega]-C_{q q}[-\omega]=l_{P}^{2} \frac{b[\omega]}{\omega}
$$

( $b$ is an even function). Note that this commutator, computed here in the vacuum state (of gravity waves), is state-independent. The same value would be obtained in a stochastic background of gravity waves (at the level of approximation considered in this letter).

The commutator between the distance variation $q$ and the associated velocity $q^{\prime}$ (which is also the redshift parameter) is thus directly related to the function $b$ :

$$
C_{q^{\prime} q}[\omega]-C_{q q^{\prime}}[-\omega]=i l_{P}^{2} b[\omega]
$$

Translating back to the time domain provides us with commutators describing quantum fluctuations of geodesic distances:

$$
\begin{aligned}
{\left[q^{\prime}(t), q(0)\right] } & =-i l_{P}^{2} b(t) \\
{[q(t), q(0)] } & =-i l_{P}^{2} B(t) \\
B(t) & =\int_{0}^{t} b\left(t^{\prime}\right) d t^{\prime}
\end{aligned}
$$

Explicit expressions of these functions are obtained for one-way tracking:

$$
\begin{aligned}
b^{(1)}(t) & =\frac{8}{3} \delta(t)-\frac{(2 \tau-|t|)^{2}}{2 \tau^{3}} \theta(2 \tau-|t|) \\
B^{(1)}(t) & =\varepsilon(t) \frac{(2 \tau-|t|)^{3}}{6 \tau^{3}} \theta(2 \tau-|t|)
\end{aligned}
$$

and for two-way tracking:

$$
\begin{aligned}
b^{(2)}(t) & =\delta(t)-\frac{1}{6}(\delta(t-2 \tau)+\delta(t+2 \tau))+\frac{(|t|-\tau)(2 \tau-|t|)}{2 \tau^{3}} \theta(2 \tau-|t|) \\
B^{(2)}(t) & =\varepsilon(t) \frac{-2|t|^{3}+9|t|^{2} \tau-12|t| \tau^{2}+6 \tau^{3}}{12 \tau^{3}} \theta(2 \tau-|t|)
\end{aligned}
$$

The short-time dynamics is independent of the mean propagation time (as the high-frequency limit). A remarkable property of the commutator is that it contains a step at null delay whose size is given by the high-frequency limit $b[\infty]$ of the function $b$ :

$$
B(t) \approx \frac{1}{2} b[\infty] \varepsilon(t) \quad(|t| \ll \tau)
$$

\section{DISCUSSION}

Quantum fluctuations of gravitational fields set a limit for sensitivity in length measurements. This limit corresponds to irreducible fluctuations for geodesic distances, which have a quantum character even in empty space. It follows that a consistent treatment of vacuum fluctuations and gravitation must take into account the non-commutative quantum geometry of space-time [21].

The quantitative expressions (8) (or (9)) obtained for the gravitational quantum limit have an order of magnitude mainly determined by Planck length. This is consistent with the often-expressed idea (see for instance [6]) that Planck length limits the range of validity of a classical description of space-time. Let us however emphasize that we have characterized the space-time fluctuations for noise frequencies much smaller than Planck frequency. As a consequence, we can study their (small) effects in the domain of experimentally accessible frequencies, although we do not yet have at our disposal a complete theory of Quantum Gravity (which would describe their large effects at the Planck frequency). In particular, we can consider the possibility of (small) modifications in the effective (low-frequency) theory of gravitation, or of quantum fields. 
An interesting conclusion about the significance of Planck mass emerges from a comparison between gravitational quantum limit (8) and vacuum-pressure quantum limit (5). They have rather similar forms if we consider as unimportant the explicit forms of the dimensionless functions $\Phi$ and $b$. The main difference between them is that their orders of magnitude are determined respectively by Planck length $l_{P}$ and Compton wavelength $\lambda_{C}$. It follows that vacuum-pressure fluctuations dominate for masses smaller than Planck mass while gravitational quantum fluctuations dominate for masses greater than Planck mass.

At this point, it is worth emphasizing that Planck mass does not appear such an unaccessible value as Planck length or Planck frequency (see eqs (1)). Remarkably enough, Planck mass lies near the borderline between microscopic and macroscopic masses. It is thus tempting to regard this property, not as an accidental coincidence, but as a consequence for masses greater than Planck mass of the dominance of a universal fluctuation mechanism associated with gravitational vacuum fluctuations [22].

Concerning the theory of measurement, it appears that an apparatus designed for length measurement is able (in an analysis restricted to questions of principle and if the measurement strategy is optimized) to register the proper quantum fluctuations of geodesic distances only if it is built with masses greater than Planck mass. Otherwise, it adds fluctuations of its own which are greater than geodesic fluctuations by a ratio $\frac{m_{P}}{m}$. It may be stated in other words that, when quantum fluctuations (and not only classical trajectories) are considered, macroscopic masses (but not microscopic ones) are found to obey the principle of universality of free fall.

[1] Salecker H. and Wigner E.P., Physical Review 109571 (1958).

[2] Caves C.M.Physical Review Letters 4575 (1980); Physical Review D23 1693 (1981).

[3] Unruh W.G. in "Quantum Optics, Experimental Gravitation and Measurement Theory" eds P.Meystre and M.O.Scully (Plenum, 1983), p.647.

[4] Jaekel M.T. and Reynaud S., EuroPhysics Letters 13301 (1990); Pace A.F., Collett M.J. and Walls D.F., Physical Review A47 3173 (1993).

[5] Jaekel M.T. and Reynaud S., Journal de Physique I-3 1 (1993).

[6] Wheeler J.A., Annals of Physics 2604 (1957); DeWitt B.S., in "Gravitation, An Introduction to Current Research" ed. L.Witten (Wiley, 1962), p.266; v. Borzeszkowski H.H. and Treder H.J., Annalen der Physik 40287 (1983); Padmanabhan T., Classical and Quantum Gravity 4 L107 (1987).

[7] Mashhoon B. and Grishchuk L.P., Astrophysical Journal 236990 (1980); Hellings R.W. in "Detection of Gravitational Waves" ed. D. Blair (Cambridge University Press, 1992), p.453.

[8] Grishchuk L.P., Uspekhi Fizika Nauk 121629 (1977) [Sov. Phys. Usp. 20319 (1977)]; Grishchuk L.P., Uspekhi Fizika Nauk 156297 (1988) [Sov. Phys. Usp. 31940 (1988)].

[9] Gupta S.N., Proceedings of the Physical Society A65 11, 40 (1952); Feynman R.P., Acta Physica Polonica 24697 (1963).

[10] Caves C.M., Thorne K.S., Drever R.W.P., Sandberg V.D. and Zimmermann M, Reviews of Modern Physics 52 341 (1980).

[11] Yuen H.P. Physical Review Letters 51719 (1983); Caves C.M. Physical Review Letters 542465 (1985); Ozawa M. Physical Review Letters 60385 (1988); Ozawa M. Physical Review A41 1735 (1990).

[12] Reynaud S., Heidmann A., Giacobino E. and Fabre C., in "Progress in Optics XXX" ed. E.Wolf (1992), p.1.

[13] Braginsky V.B. and Khalili F.Ya., "Quantum Measurement" (Cambridge, 1992).

[14] Jaekel M.T. and Reynaud S., Quantum Optics 439 (1992).

[15] Barton G., Journal of Physics A24 991, 5533 (1991); Maîa Neto P.A. and Reynaud S., Physical Review A47 1639 (1993); Barton G., in "Cavity Quantum Electrodynamics" (Supplement: Advances in Atomic, Molecular and Optical Physics), P.Berman ed., (AcademicPress, 1994).

[16] Braginski V.B. and Khalili F.Ya. Physics Letters A161 197 (1991); Jaekel M.T. and Reynaud S., Journal de Physique I-2 149 (1992).

[17] Jaekel M.T. and Reynaud S., Annalen der Physik 468 (1995).

[18] Misner C.W., Thorne K.S. and Wheeler J.A., "Gravitation" (Freeman and Company, 1973); see also Braginskii V.B. and Menskii M.B., Pisma Zh. Eksp. Teor. Fiz. 13585 (1971) [Soviet Physics JETP Letters 13417 (1971)].

[19] Sachs R.K. and Wolfe A.M., Astrophysical Journal 14773 (1967).

[20] Braginsky V.B., Kardashev N.S., Polnarev A.G. and Novikov I.D., Nuovo Cimento 105B 1141 (1990).

[21] Connes A. "Non commutative differential geometry" (IHES, Extrait des Publications Mathématiques 62, 1986); Woronowicz S.L., Communications in Mathematical Physics 122125 (1989).

[22] Rosen N., American Journal of Physics 32597 (1964); Karolyhazy F., Nuovo Cimento 42A 390 (1966). 